\title{
Complexité de la famille des facteurs de croissance FGF : la preuve par 9
}

Les interactions qui régissent la prolifération et la différenciation cellulaires sont, on le sait, complexes, mais on ne peut s'empêcher de s'étonner encore devant le nombre sans cesse croissant de molécules de signalisation, telles que les cytokines, neuropeptides et autres facteurs de croissance, nécessaires à la régulation d'un organisme de mammifère. Chaque famille de ces protéines enregistre régulièrement la découverte de nouveaux membres. C'est le cas de la famille des FGF (anciennement fibroblast growth factors), dont deux membres viennent tout juste de recevoir le baptême : FGF8 et FGF9. La reconnaissance de la famille s'est faite schématiquement en trois étapes. Il y eut d'abord la caractérisation, au début des années 1980, des gènes des deux membres "fondateurs" de la famille, $F G F 1 / a F G F$ et $F G F 2 / b F G F$, puis un accroissement brutal lorsque le nombre des gènes $F G F$ caractérisés est passé à sept, principalement sous l'impulsion d'études sur les oncogènes auxquels les FGF sont directement apparentés $[1,2]$. La taille connue de la famille est ensuite longtemps restée stationnaire, si bien que l'on croyait en connaître tous les membres. L'identification presque simultanée de FGF8 [3] et FGF9 [4] après ce temps de latence oblige à reconsidérer la question, déjà difficile, de la complexité des FGF.

Une réelle communauté de structure

L'alignement et la comparaison des séquences protéiques des 9 FGF (figure 1) permet d'en faire ressortir les similitudes, de dériver un dendrogramme (figure 2) et ainsi de représenter leurs parentés potentielles. Les ressemblances sont concentrées dans une région noyau de 130 acides aminés environ, laissant des extrémités amino- et carboxy-terminales large$\mathrm{m} / \mathrm{s} n^{\circ} 10$ vol. 9 , octobre 93 ment divergentes. Bien que réelles, les similitudes de séquence entre FGF sont, somme toutes, assez limitées : si l'on excepte les paires FGF1/FGF2 et FGF4/FGF6 qui présentent respectivement $53 \%$ et $70 \%$ de positions identiques (Tableau I), ce score se situe aux alentours de $25-30 \%$, avec même un minimum à $15 \%$ d'acides aminés identiques. Pratiquement, ce faible score a exclu la possibilité de clonage de nouveaux membres par le biais de leur ressemblance, à l'exception notoire de FGF6 $[5,6]$. Ce relatif éloignement des séquences primaires des FGF cache cependant une communauté structurale bien réelle, illustrée par la communauté de récepteurs. Seuls quatre gènes codant pour des récepteurs à tyrosine kinase capables de lier les FGF et de transduire un signal mitogénique ont été décrits. Bien que chaque gène soit capable de coder plusieurs isoformes présentant des affinités sensiblement différentes pour les FGF, chaque FGF testé peut se lier à plusieurs récepteurs [7]. Il faut noter cependant que les récepteurs des FGF8 et FGF9 ne sont pas encore connus. Il a pu être montré par ailleurs que l'interleukine $1 \beta$ (IL1 $\beta$ ), qui présente des similitudes de séquence avec FGF1 et FGF2, adopte une structure tertiaire proche de la leur [8-10], mais occupe néanmoins des récepteurs totalement distincts. La redondance observée alors quant à la liaison des FGF à leurs récepteurs suggère que la ressemblance structurale est bien plus forte que ne le laissent présager les similitudes de séquences.

\section{Peut-on parler d'une communauté} de fonction?

S'il existe une réelle communauté de structure entre les FGF qui a d'importantes répercussions sur leurs propriétés, il est plus difficile d'envisager une communauté de fonction. Les FGF apparaissent plutôt comme des facteurs multifonctionnels, bien

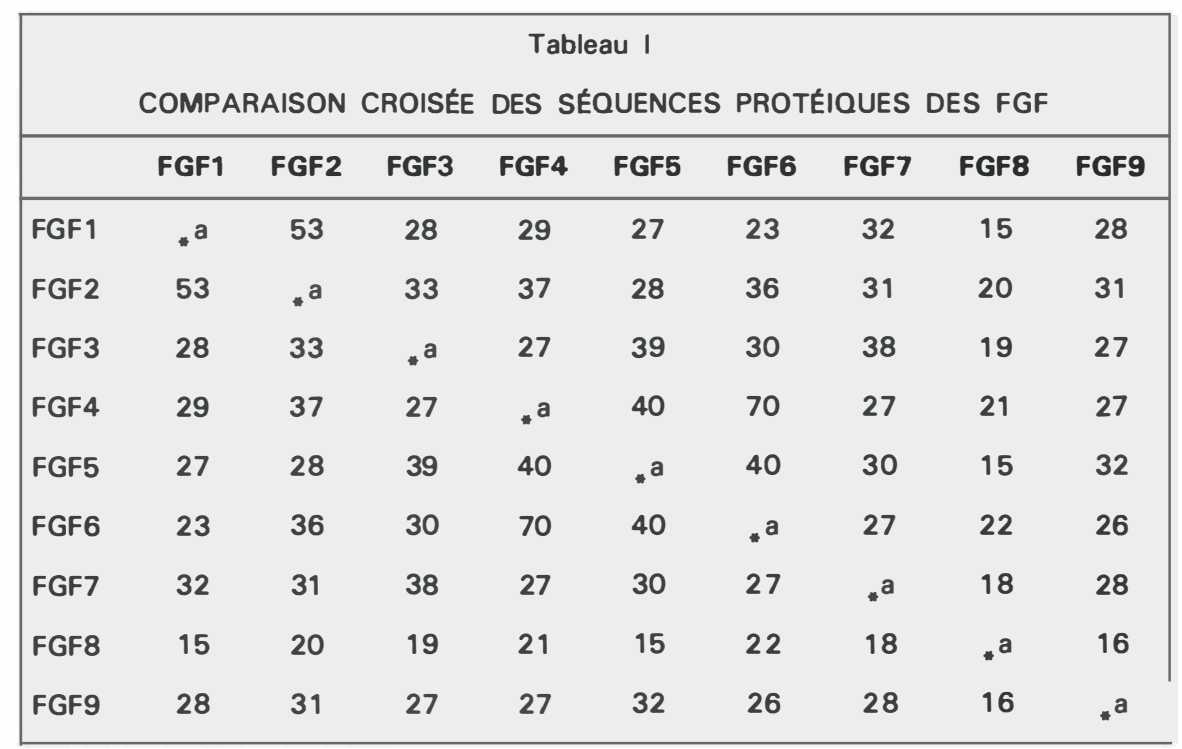

a Données non pertinentes.

Pourcentage d'acides aminés identiques dans la région cœur. 


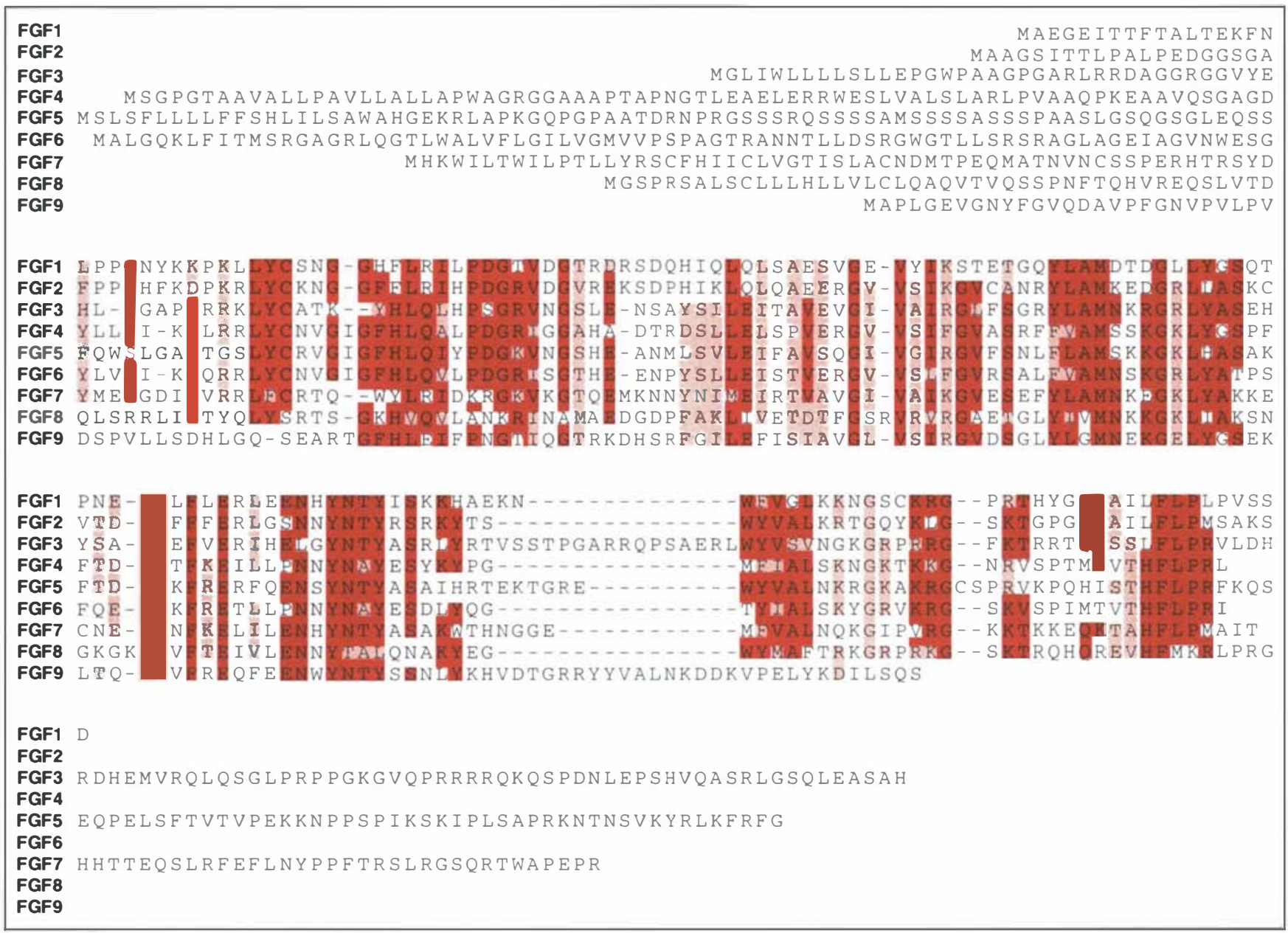

Figure 1. Comparaison des séquences protéiques des 9 FGF connus. Les séquences des protéines humaines (FGF1 à 7, FGF 9) ou murine (FGF8) ont été alignées en ménageant des interstices (-) de façon à faire ressortir les régions de similitude. Les résidus colorés en rouge ou en rose correspondent à des positions où 5 acides aminés ou plus sont respectivement identiques ou similaires. Une région cœur où les FGF présentent de fortes similitudes de séquence est évidente.

Figure 2. Arbre phylogénétique (dendrogramme) déduit des comparaisons de séquences. Les différences entre chaque séquence protéique (région cœur) ont été calculées et utilisées pour construire ce dendrogramme. Les distances horizontales sont ainsi proportionnelles au nombre de substitutions par rapport à un ancêtre commun hypothétique.

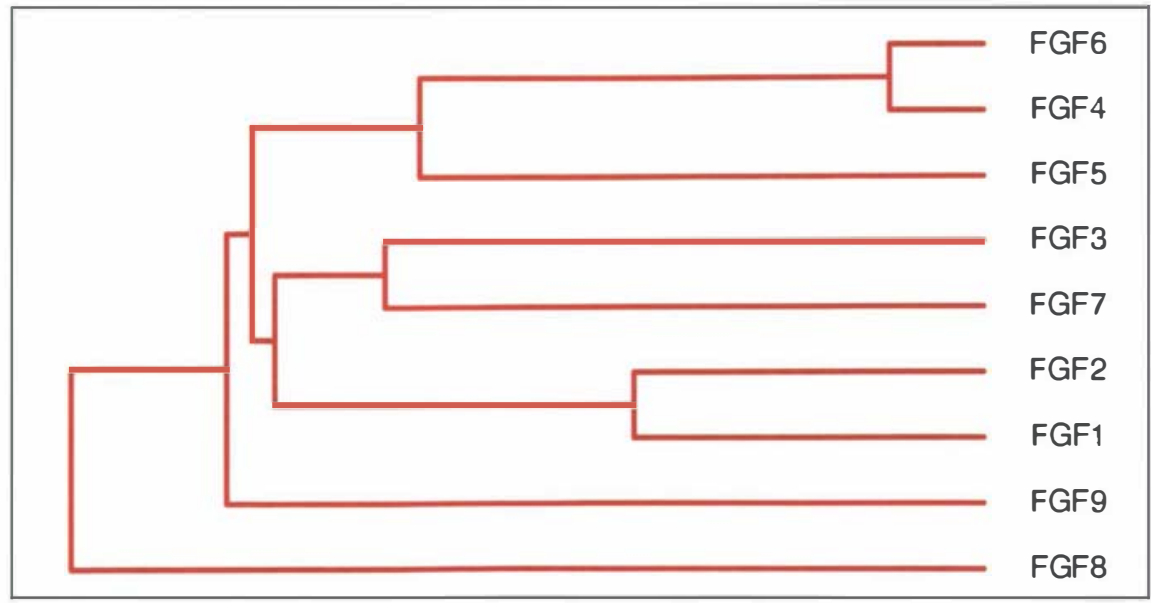


que des différences notables existent entre leurs capacités. Ainsi, par exemple, si FGF2 semble ubiquiste et capable d'agir sur de multiples types de tissus, le rôle de FGF7 semble limité aux épithéliums. La plupart des FGF semblent cependant exercer un rôle important dans l'embryogenèse, lors du développement de certains tissus [11-14]. Plusieurs FGF peuvent être exprimés dans un même tissu pendant des périodes qui se chevauchent. Un exemple en est le muscle squelettique [15]. Dans ces cas, on peut se demander si les FGF agissent à des étapes différentes, en synergie, ou encore si un certain degré de redondance est autorisé par leur communauté de structure et leurs interactions complexes avec les mêmes récepteurs. L'obtention de différentes souches de souris mutantes dans lesquelles les gènes $F G F$ auront été rendus non fonctionnels par recombinaison homologue pourra apporter des réponses à ces questions.

\section{Et maintenant ?}

L'existence d'autres FGF non identifiés est possible. La caractérisation d'une activité "facteur de croissance " à partir d'une cellule ou d'un tissu, suivie du clonage du gène, s'est révélée être une tactique efficace pour isoler de nouveaux FGF. Cependant, d'autres stratégies pourraient être utilisées. On peut, par exemple, imaginer une approche par création d'une boucle autocrine, consistant à transfecter des cellules initialement dépourvues de récepteurs par des gènes de récepteurs et des ADNc d'une librairie d'expression. Malgré cela, seuls les programmes d'étude du génome pourront répondre de manière définitive à la question du nombre de gènes FGF. Mais le difficile problème posé par l'attribution de leurs fonctions respectives sera peut-être plus long à résoudre

\section{RÉFÉRENCES}

1. Goldfarb M. The fibroblast growth factor family. Cell Growth Differ $1990 ; 1$ : 439-45.

2. Benharroch D, Birnbaum D. Biology of the fibroblast growth factor gene family. Israel J Med Sci 1990 ; 26 : 212-9.

3. Tanaka A, Miyamoto K, Minamino N, Takeda M, Sato B, Matsuo H, Matsumoto $\mathrm{K}$. Cloning and characterization of an androgen-induced growth factor essential for the androgen-dependent growth of mouse mammary carcinoma cells. Proc Natl Acad Sci USA $1992 ; 89$ : 8928-32.

4. Miyamoto M, Naruo KI, Seko C, Matsumoto $\mathrm{S}$, Kondo $\mathrm{T}$, Kurokawa $\mathrm{T}$. Molecular cloning of a novel cytokine cDNA encoding the ninth member of the fibroblast growth factor family, which has a unique secretion property. Mol Cell Biol 1993 ; 13 : 4251-9.

5. Marics I, Adélaïde J, Raybaud F, Mattei MG, Coulier F, Planche J, de Lapcyrière $\mathrm{O}$, Birnbaum $\mathrm{D}$. Characterization of the $H S T$-related $F G F 6$ gene, a new member of the fibroblast growth factor gene family. Oncogene $1989 ; 4$ : 335-40.

6. Coulier F, Pizette S, Ollendorff V, de Lapeyrière $\mathrm{O}$, Birnbaum $\mathrm{D}$. The human and mouse Fibroblast Growth Factor 6 (FGF6) genes and their products : possible implication in muscle development. Progress in Growth Factor Research 1993 (sous presse).

7. Coulier F, Pizette S, Batoz M, Birnbaum D. Les récepteurs des facteurs de croissance des fibroblastes : une famille qui s'agrandit. médecine/sciences $1992 ; 8: 811-8$.

8. Zhu X, Komiya H, Chirino A, Faham S, Fox GM, Arakawa T, Hsu BT, Rees DC. Three-dimensional structures of acidic and basic fibroblast growth factors. Science $1991 ; 251: 90-3$.

9. Zhang J, Cousens LS, Barr PJ, Sprang SR. Three-dimensional structure of human basic fibroblast growth factor, a structural homolog of interleukin 1 $\beta$. Proc Natl Acad Sa USA 1991; 88 : 3446-50.

10. Eriksson AE, Cousens LS, Weaver LH, Matthews BW. Three-dimensional structure of human basic fibroblast growth factor. Proc Natl Acad Sci USA 1991; 88 : 3441-5.

11. Niswander L, Martin GR. Fgf-4 expression during gastrulation, myogenesis, limb and tooth development in the mouse. Development $1992 ; 114$ : 755-68.

\section{TIRÉS A PART}

F. Coulier.

12. Haub O, Goldfarb M. Expression of the fibroblast growth factor-5 gene in the mouse cmbryo. Development 1991; 112 : 397-406.
13. Represa J, León Y, Miner C, Giraldez F. The int-2 proto-oncogene is responsible for induction of the inner ear. Nature 1991 ; $353: 561-3$.

14. Mansour SL, Goddard JM, Capecchi MR. Mice homozygous for a targeted disruption of the proto-oncogene int-2 have developmental defects in the tail and inner ear. Development 1993 ; 117 : 13-28.

15. de Lapeyrière $\mathrm{O}$, Ollendorff $\mathrm{V}$, Planche J, Ott MO, Pizette S, Coulier F, Birnbaum D. Expression of the Fgf6 gene is restricted to developing skeletal muscle in the mouse embryo. Development 1993; 118 : 601-11.

\section{François Coulier}

Chargé de recherche à l'Inserm.

Odile de Lapeyrière

Chargée de recherche à l'Inserm.

Daniel Birnbaum

Directeur de recherche à l'Inserm. Laboratoire d'oncologie moléculaire, Inserm U. 119, 27, boulevard Leï Roure, 13009 Marseille, France. 